\title{
Small-Scale Multiaxial Setup for Damage Detection Into the Very High Cycle Fatigue Regime
}

\author{
T. Straub ${ }^{1,2}$ - M.F. Berwind ${ }^{2,5}$ - T. Kennerknecht ${ }^{2} \cdot$ Y. Lapusta $^{3}$ - C. Eberl $^{2,4,5}$
}

Received: 28 June 2014 / Accepted: 9 April 2015 /Published online: 12 May 2015

(C) The Author(s) 2015. This article is published with open access at Springerlink.com

\begin{abstract}
Micro electro mechanical systems, coatings, and thin films contain materials that are often subjected to complex loading conditions and high frequencies. Therefore, the implementation of a resonant multiaxial fatigue setup for small-scale samples is of high interest. Finite Element simulations have been used to design small-scale samples which possess very close bending and torsional resonant frequencies. The bending and torsional modes are excited by two piezo actuators working either in or out of phase with one another. The bending and torsional amplitudes are measured independently by a laser and the actuator amplitudes are controlled by a Field Programmable Gate Array. The fatigue setup can be used with a varying range of sample sizes from centimeters down to tens of micrometers. The novel multiaxial resonant micro fatigue setup as well as results (fatigue damage for $\mathrm{Ni}$ and lifetime for $\mathrm{Cu}$ ) from bending small-scale fatigue tests are shown and discussed.
\end{abstract}

Keywords Nickel - Copper · Face centered cubic metals . Multiaxial $\cdot$ Small-scale $\cdot$ Resonant fatigue setup $\cdot$ Very high cycle fatigue $\cdot$ Persistent slip bands $\cdot$ Crack initiation $\cdot$ Crack propagation

\section{T. Straub}

thomas.straub@iwm.fraunhofer.de

1 Institute for Applied Materials (IAM), Karlsruhe Institute of Technology (KIT), 76021 Karlsruhe, Germany

2 Fraunhofer Institute for Mechanics of Materials (IWM), Wöhlerstr. 11, 79108 Freiburg, Germany

3 French Institute of Advanced Mechanics (IFMA-LAMI), Campus de Clermont-Ferrand/Les Cezeaux, 63175 Aubière, France

4 Department of Mechanical Engineering, Johns Hopkins University, Baltimore, MD, USA

5 Institute for Micro Systems Technology, Albert-Ludwigs University, 79110 Freiburg, Germany

\section{Introduction}

Small-scale fatigue is relevant to the applications and research of many current material science and engineering topics, such as thin films [1], micro electro mechanical systems (MEMS) [2], surface acoustic wave devices [3] or microelectronics. Such systems are typically constrained by their complex structure and often work at particularly high frequencies of up to several $\mathrm{GHz}[4,5]$. Therefore, required lifetimes are in the high (until $10^{7}$ ) and very high (beyond $10^{8}$ ) cycle regime, while cyclic loading conditions can be multiaxial and lead to damage accumulation until failure.

Recent experiments on body-centered cubic metals [6] explored the very high cycle fatigue (VHCF) domain beyond $10^{7}$ cycles and distinguish two regimes e.g., for high strength steels. The lower regime corresponds to the transition from the high cycle fatigue (HCF) limit (e.g., $10^{7}$ cycles) to the VHCF regime (e.g., $10^{9}$ cycles) [7-10]. In this domain, a surface roughening can be observed due to irreversible cyclic slip of dislocations. The fourth regime represents the VHCF limit, below which cyclic slip would become essentially reversible and non-damaging. For pure face-centered cubic metals it is still unclear if there is a fatigue limit similar to such a regime. Al thin films do not show such a limit for up to $10^{14}$ cycles and a stress amplitude of roughly $14 \mathrm{MPa}$ [5]. One such experiment was carried out at $1 \mathrm{GHz}$ and the authors argue that at such high frequencies the fatigue mechanisms are constrained and can differ from low frequency fatigue [3]. Nevertheless, it has been shown for pure copper that the lifetime in the VHCF regime is controlled by crack initiation as a consequence of irreversible plastic deformation at the surface, such as slip markings [10-14]. The slip markings can lead to the initiation of micro cracks, where one of them may undergo continued short crack growth, followed by fatal failure. In the case of VHCF, the lifetime is mostly determined by crack initiation followed by micro and short crack growth, while the macroscopic crack growth is only a small part of the total lifetime [15]. Therefore, crack growth experiments are important for tracking damage evolution, since grain size and 
orientation have important implications on the topic [16]. For bulk samples, VHCF experiments are typically carried out at much lower frequencies of $20 \mathrm{kHz}$ with resonant fatigue setups. Commonly such setups induce a fully reversed uniaxial stress state [17]. An ultrasonic fatigue setup for torsional fatigue testing was implemented by the group of Bathias [18].

But not only the frequency and the microstructure can have an effect on lifetime, the sample size also plays a role. Furuya showed for high strength steel bulk samples that the mean VHCF lifetime increases by an order of magnitude with decreasing specimen volume from $\sim 800$ to $\sim 30 \mathrm{~mm}^{3}$ [19]. This is known as a classical size effect which can set in when the tested samples are too small to represent the microstructural feature and defect distribution. Following a random distribution, the largest defects typically exist only in limited numbers within smaller samples, and more negatively impact the lifetime of the sample compared to smaller defects. As a result, the mean lifetime of smaller samples seems to increase, while generally, for all sample dimensions, the trend is toward an increasing scatter at higher lifetimes.

For very small dimensions, as in thin films, MEMS or nanostructured materials, the damage mechanisms can be constrained or even restricted. Here, the extrinsic and intrinsic dimensions approach the size of the damage-governing mechanisms and processes, e.g., dislocations and dislocation structures. This has been observed for metal thin films, where film thickness is inversely proportional to fatigue strength [20]. The microstructure of such films is smaller than typical fatigue structures (e.g., persistent slip bands). Thus, such characteristic fatigue structures cannot develop. Although the size constraining effects on mechanical properties or fatigue behavior are not fully understood, such materials are used in safety relevant systems. Furthermore, little research has been conducted on small-scale multiaxial fatigue while this is the typical loading condition in a real environment. Therefore, a novel small-scale multiaxial fatigue setup has been designed and implemented.

This manuscript is structured into two main parts. The working principle, simulation and implementation are presented in the first part. Thereafter, fatigue damage results on $\mathrm{Ni}$ and lifetime results on $\mathrm{Cu}$ small-scale samples are presented.

\section{Custom Built Resonant Multiaxial Micro Fatigue Setup}

\section{Experimental Principle}

Multiaxial fatigue experiments with micro samples at $\mathrm{kHz}$ frequencies require novel experimental setups. For bulk samples and MEMS devices, such apparatus are typically designed as resonant devices and can only be used at their characteristic frequencies to maximize the stress amplitude. In the here presented novel experimental setup such a principle is implemented as well.

The initial approach is to fix the sample on one side with a clamp and to displace this clamp by two independently controlled piezo actuators normal to the sample surface (P1 and $\mathrm{P} 2$ ). In phase, a cyclic actuation at P1 and P2 leads to a bending vibration of the sample (Fig. 1(a)), while an anti-phase excitation at $\mathrm{P} 1$ and $\mathrm{P} 2$ leads to a torsional vibration (Fig. 1(c)). A combined signal leads to a multiaxial excitation, a combination of bending and torsional oscillations.

\section{Sample Design and Finite Element Simulations}

Two kinds of Finite Element (FE) analyses were conducted. The static analysis allowed an estimation of the stress distribution in the sample under a certain deformation. In this case an out of plane displacement $U y$ was applied to simulate bending, with an angular rotation $\alpha$ of the mass around the $\mathrm{Z}$ axis of the sample to simulate a torsional deformation. The second analysis was modal in nature, and is typically used to determine the resonant modes of the model. It can also serve as a first step for more complex analyses such as the harmonic response of the sample or a full transient dynamic analysis. Furthermore, the analysis is a necessary input for multiaxial fatigue models.

In both simulations, all displacement degrees of freedom were set to zero at the two gripping surfaces (Fig. 2). In the static analysis, bending was simulated with an out of plane displacement in the y direction applied on the nodes at the inner edge of the mass (going through point P, Fig. 2). Torsion was simulated with an angular rotation $\alpha$ of the mass around the $\mathrm{Z}$ axis of the sample. Therefore, all nodes on the same line were displaced by a linearly changing amount, $U y$, being zero at point $P$ (Fig. 2). In this case, the displacement depends on the coordinate $X \mathrm{n}$ of each node $\mathrm{n}: U y=\tan (\alpha) \times X \mathrm{n}$. In the modal analysis, the gripping section is also fixed but no further boundary conditions are applied.

To conserve computation time, the sample has been cut into three parts: the mass and the gripping section with the same coarse element size, and the double-filleted gage section with a somewhat smaller element size. The element sizes were chosen based on a compromise between the convergence of the results and a reasonable computation time. In the gage section, two elements were used through the sample thickness of $200 \mu \mathrm{m}$. For the gripping and mass sections, a single hexahedral element was sufficient to model the sample thickness. With these element sizes and meshing the sample with an automatic sweep mesh, the resonant frequency value converges with a $0.1 \%$ error.

All simulations have been carried out with ANSYS using various mesh elements, and SOLID 185 was chosen as the best option. SOLID 185 is used for 3-D modeling of solid structures, and is defined by eight nodes having three degrees 


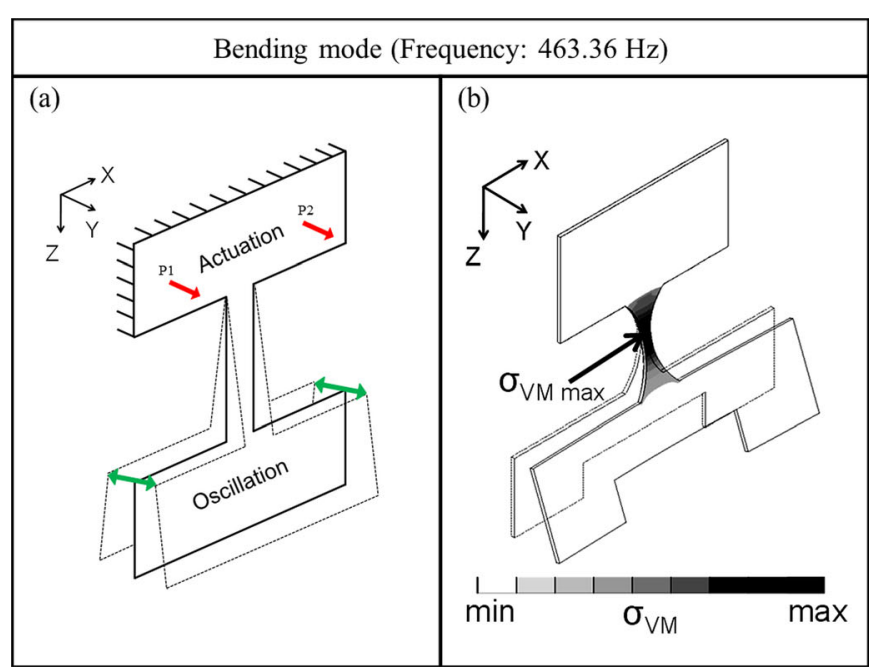

Fig. 1 Schematics representing the actuation and oscillation for the bending (a) and torsional (c) modes. (Locations of actuation by the two piezo actuators marked with arrows at P1 and P2). And finite element
Torsional mode (Frequency: $454.34 \mathrm{~Hz}$ )

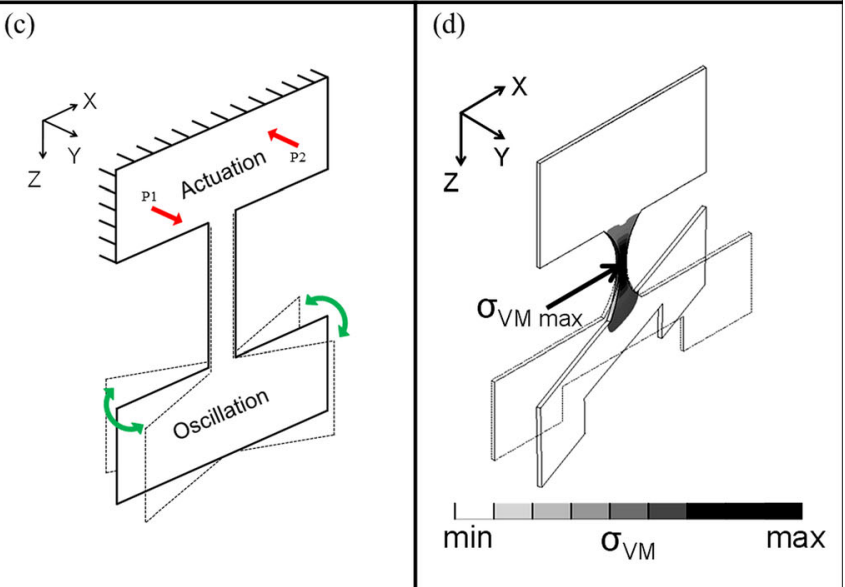

modal analysis showing the maximum stress in the gage section of the samples for the bending (b) and torsional (d) mode

of freedom at each node. This element type allows a smooth transition between volumes with different mesh sizes (in our case, the gripping and mass sections have a single element along the thickness while the gage section has two elements). With this element, the stress extraction is possible at each node and allows having comparable node and element solutions, which are converging to the same value due to the previously explained FE conditions. Finally, each sample material has isotropic elastic properties.

When a fatigue sample is designed to work at its resonance, the sample geometry must be optimized considering restrictions from the setup. To achieve multiaxial loading conditions, the sample needs to be designed in a way that the torsion and bending modes do have a similar resonant frequency. The resonant frequency of the sample is defined by the stiffness $k$ of the gage section and the attached mass $m$ at the free end:

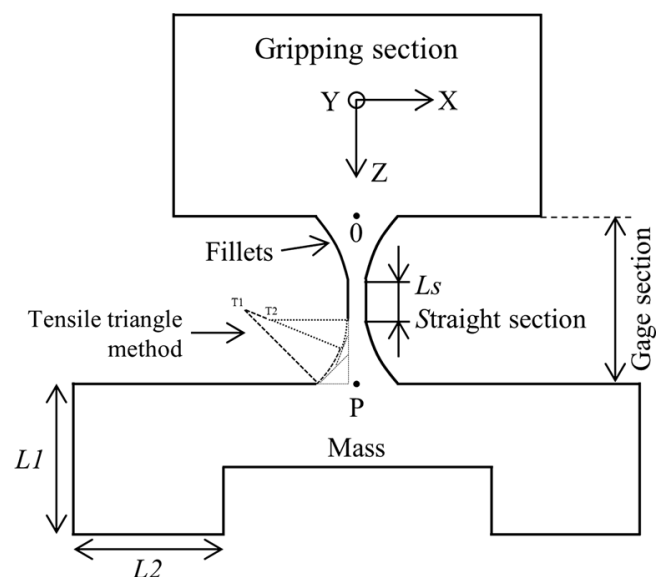

Fig. 2 Sample geometry consisting of gripping section, gage section and mass. The sample thickness is uniform. The optimization of the fillets has been made with the tensile triangle method [21]. T1 and T2 represent the center of the two arcs

$f \propto \sqrt{k / m}$. In order to define the resonant frequency in torsion and bending independently, the mass at the end of the sample was chosen to be U-shaped (Fig. 2). For a cantilever beam of length $l$ which is fixed on one end and carries a point mass $m$ on the other end, the resonant frequency is represented by $f=1 /(2 \pi) \sqrt{3 \mathrm{EI} /\left(m l^{3}\right)}$ where $\mathrm{E}$ is the Young's modulus, and I the moment of inertia. Thus, for the simulated sample, the distance of the center of mass from the gripping section (comparable to 1) has a large impact on the resonant frequency. This distance is related to the modeling parameter $L 1$. In the following, the design and optimization of such samples is briefly introduced. The samples, can be divided into three sections:

1. The gripping section, which is settled into the clamps of the setup. The design requires a firm grip which does not introduce friction at the gripping section which would influence the measurement.

2. The gage section, which has a length of $5 \mathrm{~mm}$. The shoulder fillets have been optimized using C. Mattheck's method of tensile triangles [21]. The aim is to reduce the notch stresses where the fillet joins the straight gage section. Such localized stress concentrations can lead to the initiation of cracks and failure. Due to the bio-inspired Mattheck geometric optimization, it is possible to reduce these stresses at the notch by deriving the fillet with the usage of a least three stacked triangles, starting with the largest radius $(4.89 \mathrm{~mm})$ at the fixation, and ending with the smallest radius $(3.49 \mathrm{~mm}$ ) joining the gage section (Fig. 1(b) and (d)). The straight section with a length $L s$ is situated between the lower and the upper shoulder fillets. Two gage section geometries with the same cross section were simulated. The first has a straight section of $L s=500 \mu \mathrm{m}$ and the second an hourglass shape with 
$L s=0 \mu \mathrm{m}$. The two simulations show the maximum bending and torsional stresses at the same point at the end of the two lowest fillets.

3. Together with the stiffness of the gage section, the geometry of the mass defines the resonant frequencies of the sample. Therefore, an optimization has been conducted by the help of an FE analysis (ANSYS ${ }^{\circledR}$, USA) and MATLAB mathematical software (Mathworks ${ }^{\circledR}$, USA) to find the length $L 1$ and the width $L 2$ of the two rectangles (Fig. 2). These parameters define the two resonant modes (bending and torsion) of the sample at frequencies very close to each other. The length $L 1$ and the width $L 2$, (Fig. 2), of the two rectangles were equally varied in order to find the frequency at which the bending and torsional modes cross. Changing $L 2$ does not change the width of the sample, but only the width of the rectangles. For the proof of concept, Ni samples with a thickness of $200 \mu \mathrm{m}$ were designed and the results are shown in Fig. 3(a). The point at which the bending and torsion modes cross was found to be at $460 \mathrm{~Hz}$ for a length of $L=L 1=L 2=4.5 \mathrm{~mm}$. The disadvantage of this design is that the resonant frequency is rather low (Fig. 3(a)), and more than 25 days would be necessary to reach $10^{9}$ cycles. Therefore, consecutive optimizations were performed with the goal of reaching the $\mathrm{kHz}$ regime. The geometry of the mass plays an important role on the resulting resonant frequencies of the sample. With an increase in the length $L 1$ of the mass, a decrease in the bending mode resonant frequency can be expected. $L 1$ also has an impact on the torsional resonant frequency, but it is significantly lower. In the same way, the larger the width $L 2$, the lower the torsional frequency is, resulting in a minor change in the bending frequency. Nevertheless, both modes are influenced by the total mass and the heavier the mass, the lower is the resonant frequency. Based on this behavior, an optimization has been conducted by the help of an FE modal analysis (ANSYS) to find the length $L 1$ and the width $L 2$ of the two rectangles which leads to achieve high frequencies for the resonant modes (bending and torsion) with close proximity. In case of $L 1=L 2=L$, the simulation shows that the torsional mode frequency decreases faster than the bending mode frequency when $L$ increases. Therefore $L 1$ and $L 2$ are defined such as: $L 1=x . L$ with $x>1$ and $L 2=L$. For each value of $x$, there is one value of the length $L$ where the torsional mode frequency and the bending mode frequency are nearly equal. By plotting the multiaxial mode frequency over $L$, the mass geometry can be found for a designed resonant frequency (Fig. 3(b)). A second design, in this case for $\mathrm{Cu}$ samples, was optimized to reach a resonant frequency in the $\mathrm{kHz}$ regime. This new $200 \mu \mathrm{m}$ thick geometry has the following dimensions: $L 1=8 . L=6.32 \mathrm{~mm}, L 2=L=0.79 \mathrm{~mm}, f_{\text {multiaxial }}=1180 \mathrm{~Hz}$ and has been used for VHCF testing.
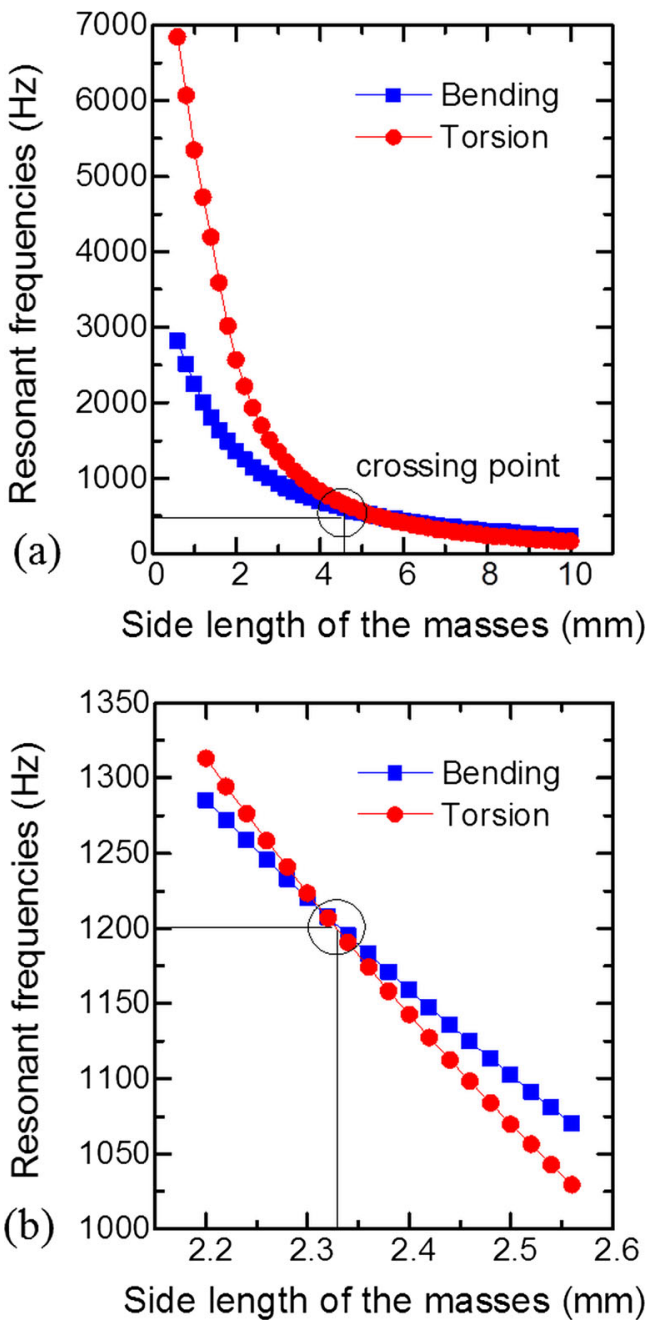

Fig. 3 FE optimization of the mass of a Ni sample where (a) $L=L 1=L 2$ and (b) $L=L 1 \neq L 2$ - resonant frequencies of bending and torsion modes are equal at the crossing point

\section{Custom Built Resonant Fatigue Setup}

A novel custom built micro fatigue setup was developed to investigate the fatigue behavior of micro samples under cyclic multiaxial load in the VHCF regime. The setup works at resonance of the sample to achieve the required excitation amplitudes. The design allows the fatiguing of micro samples in torsional and bending modes. Typically, the sample geometry (Fig. 2) is optimized by FE simulations in a way that the resonant frequencies of the torsional and bending modes are similar. Thus, it is possible to cycle the sample under a multiaxial load by choosing a driving frequency between these two resonant modes.

The design of the resonant micro fatigue setup (Fig. 4(a)) consists of two piezo actuators whereupon a flat spring is clamped. The actuation is implemented by two piezo stacks (P-843.40, Physik Instrumente, Germany), each one with a travel range of $60 \mu \mathrm{m}$, and a resolution of $1.2 \mathrm{~nm}$ in closed loop. These piezo translators are high resolution linear 

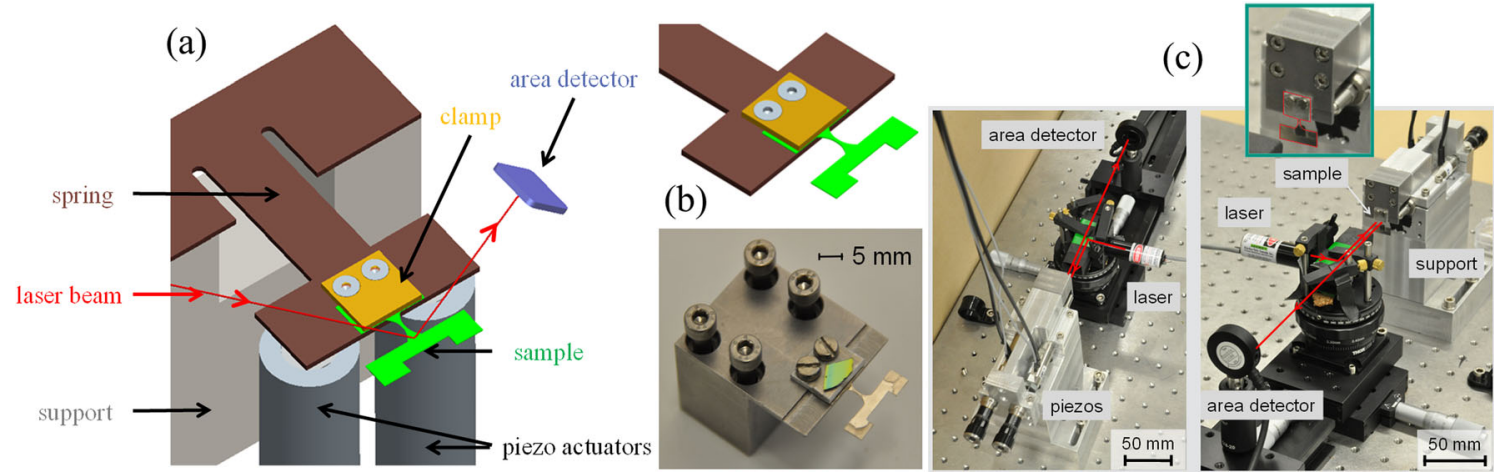

Fig. 4 (a) CAD design of the multiaxial resonant micro fatigue setup, (b) details of sample and clamp, (c) picture of the setup

actuators intended for static and dynamic applications. They provide the sub-millisecond response time necessary for $\mathrm{kHz}$ actuation. Each piezo actuator is equipped with integrated high-resolution position sensors for high precision (linearity to $0.01 \%$, and a bandwidth up to $5 \mathrm{kHz}$ ). These sensors allow measuring the two piezo displacement signals, i.e., the exact setup input actuation. The spring can be slightly bent and/or twisted by the two actuators. It is made out of steel and its geometry was optimized with FE, and chosen in a way that the surface strain is always well below the fatigue limit while its resonant frequency is well above the sample resonant frequency. With this optimization, fatigue phenomena never occur in the spring. The sample is fixed to the end of the spring and is displaced out of plane at its fixed end during bending actuation (this can be achieved by an in-phase excitation of the two piezo actuators). Inertial forces, at the sample free end, where the mass is concentrated, induce the desired bending mode in the sample. When the spring is subjected to an out-of-phase excitation of the piezo actuators, the sample oscillates around its main $\mathrm{Z}$ axis (Fig. 2) at the fixed end and a torsional mode is induced. The displacement amplitude is measured by the use of a laser beam. The beam is reflected from the surface of the sample onto an area detector. The area detector is a Position Sensitive Device (PSD), and has a high frequency analog data acquisition, an update rate up to $30 \mathrm{kHz}$, and an optical resolution of $1 \mu \mathrm{m}$. This resolution corresponds to a stress amplitude measurement error of $0.1 \%$ when considering a nickel sample in the described setup. The sensor measures the bending and torsion deflection of the sample; i.e., the exact output oscillation.

The experimental control and data acquisition software was programmed in LabView (National Instruments, USA). It mainly runs on a Field Programmable Gate Array (FPGA) (NI PCIe7852R, National Instrument, USA) which can digitize and process data from eight analog inputs at a sample rate of up to $750 \mathrm{kHz}$ (analog input $750 \mathrm{kSample} / \mathrm{s}$ and analog output $1 \mathrm{M}$ Sample/s, clock rate $40 \mathrm{MHz}$ ). Hence, the experiments can be stopped automatically in $\mathrm{ms}$ when the fatigue criterion, e.g., a frequency decrease, is exceeded. Thus, high speed load control and data acquisition are both capabilities of the setup. As an example, the two piezo actuators are controlled by the FPGA following this way: $A O_{0}=A_{1} \sin (2 \pi f t)+\left(-A_{2}\right) \sin (2 \pi f t-\theta)$ and $A O_{1}=A_{1} \sin (2 \pi f t)+A_{2} \sin (2 \pi f t-\theta)$. With $A O_{i}$ being the analog outputs from the FPGA, which are the control voltages for the piezo actuators; $A_{1}$ is the bending amplification parameter; $A_{2}$ is the torsion amplification parameter, $f$ the driving frequency in $\mathrm{Hz}, t$ the time in $\mathrm{s}$, and $\theta$ the phase angle between bending and torsion actuations. If $A_{2}=0, A O_{0}$ and $A O_{1}$ are equal, the two piezo actuators are working in phase. It has a high influence on the bending mode of the sample. On the other hand, if $A_{1}=0, A O_{0}$ and $A O_{1}$ are additive inverse, the two piezo actuators are out of phase. The sample can reach its torsion mode. If $A_{0} \neq 0$ and $A_{2} \neq 0$, the combination of bending and torsion results in a so called multiaxial mode.

Every $500 \mathrm{~ms}$, the displacement data is analyzed (tone measurement) for the amplitude of the dominating frequencies in bending and torsion, as well as the phase angles between the two modes and the two actuators. From this analysis the sinusoidal excited displacement amplitude are adjusted by a PID control. The parameters $\mathrm{P}$, I, and D were found through the application of the Ziegler-Nichols (ZN) and TyreusLuyben (TL) tuning methods. The necessary procedure to find the right PID parameters is described in the chapter 'Experimental Implementation'.

The amplitude PID and frequency controllers implemented in Labview enable amplitude and frequency control of the piezo driven excitations that occur in the sample, allowing multiaxial fatigue at resonance. The entire control process speaks of two key phase angles: $\theta$ between the two actuation modes, and $\Phi$ between the piezo actuation and sample oscillation. Controlling $\theta$ allows to change the phase shift between the torsion and bending mode. The sample phase shift, $\Phi$, is calculated from an actuation frequency signal and a displacement measurement made by the area detector. This measurement is then used as the primary plant input for the amplitude and frequency controllers. PID control regulates piezo amplitudes and thus the stress amplitude of the sample, while frequency control is responsible for keeping $\Phi$ fixed at the resonant frequency of $90^{\circ}$. Restated, the sample response measured by the surface detector acts as the primary input for 
the amplitude PID and frequency controllers; i.e., as measured displacements in the sample deviate from the desired output, the controllers regulate piezo actuation until the resonant frequency and target stress state are attained.

To calculate the stress state of the sample gage, bending and torsion deflections are measured using a laser which is reflected from the surface of the sample and is directed onto a detector. The displacement of the reflected beam on the area detector is a measure of the displacement of the sample at its free end. Therefore, it is possible to calculate the oscillation amplitude at position $P$ (Fig. 2). Finally, the displacement can be compared to a structural static finite element analysis (FEA) in order to determine the stress in the middle of the sample (Fig. 1(b) and (d)). The details of the geometric calculation for the bending mode are illustrated in Fig. 5(a). The laser is pointed onto the sample at position $P$. $Q$ is the length between "the intersection of the tangent on $P$ of the deformed sample and the non-deformed sample" and "the point $P$ from the non-deformed sample". The static and modal analyses in ANSYS show that the parameter $Q$ remained constant for a typical amplitude range applied during testing. The angle between the non-deformed sample and the tangent on point $P$ of the deformed sample is named $\alpha$. The laser beam is reflected with an angle of $2 \alpha$. Finally, $\ell_{\mathrm{b}} / 2$ is the displacement amplitude of the laser beam measured on the area detector. The same method is used for torsion. $\theta$ is the torsion angle of the sample Fig. $5(\mathrm{~b}), T$ the width of the sample and $\ell_{\mathrm{t}} / 2$ is the displacement of the laser beam measured on the area detector.

Using trigonometry and the bending and torsion deflections measured by the area detector, the real displacements $x$ (Fig. 5(a)) and $y$ (Fig. 5(b)), corresponding respectively to $\alpha$ and $\theta$ of the sample can be calculated. Due to the bending deformation, the measured displacement varies depending on the position of the laser point on the sample. In case of a large bending amplitude, the laser beam intersects with the sample at different positions depending on the deflection. However, for the typical bending amplitudes used in this paper this corresponds to an error of $0.2 \%$ which can be neglected.
The angle $\alpha$ can be calculated from the triangle formed by the tangent between the displacement $x$ at position $P$ and the length $Q$ in Fig. 5(a): $\tan (\alpha)=\frac{x}{Q}$. This angle corresponds to the deflection of the laser and the point of intersection on the detector: $\tan (2 \alpha)=\frac{\ell_{b}}{2(L-x)}$. Taking into account, that: $\tan (2 \alpha)=\frac{2 \tan (\alpha)}{1-\tan ^{2}(\alpha)}$, it can be deduced that: $\frac{\ell_{b}}{2(L-x)}=\frac{\frac{2}{Q} x}{1-\frac{1}{O^{2}} x^{2}}$ and then that $\left(\ell_{b}-4 Q\right) x^{2}+$ $4 L Q x-\ell_{b} Q^{2}=0$. The discriminant of this polynomial is: $\Delta=4 Q^{2}\left(\ell_{b}^{2}-4 \ell_{b} Q+4 L^{2}\right)$, where one solution is positive: $x=\frac{Q\left(\sqrt{\ell_{b}^{2}-4 \ell_{b} Q+4 L^{2}}-2 L\right)}{\ell_{b}-4 Q}$.

The rotational motion of the sample mass, resulting from the torsional deformation of the sample gage section, can be measured by the laser interception with the detector $y$. For pure torsion, the angle $\theta$ can be calculated on the sample side (Fig. 5(b)): $\tan (\theta)=\frac{y}{T / 2}$, and on the detector side by: $\tan$ $(2 \theta)=\frac{\ell_{\mathrm{t}}}{2 L}$. It can be deduced that: $y=\frac{T}{2} \tan (\theta)$ with $\theta=\frac{1}{2} \arctan \left(\frac{\ell_{\mathrm{t}}}{2 L}\right)$.

The deformed shape of the geometrically complex sample is analyzed by an FE modal analysis through which the variable $Q$ is calculated. A MATLAB routine is used to launch ANSYS and calculate the maximum stress in the center of the gage section depending on the displacement of the deflected laser beam $\ell_{\mathrm{t}}$ and $\ell_{\mathrm{b}}$.

Finally, the different systematic errors and uncertainties in this setup should be evaluated. The main errors stem from the determination of the sample geometry, the surface quality, and the position of the sample, as well as the measurement error of the sensors. For example, the measurement error for the sample thickness correlates linearly with the error of the calculated stress amplitude. The thickness is determined with a digital optical microscope and has a resolution of 1 pixel, corresponding to $0.18 \mu \mathrm{m}(0.09 \%$ of a $200 \mu \mathrm{m}$ thickness $)$. Furthermore, the surface of the mass section needs to be absolutely planar so that the laser beam is reflected as described in the paragraphs above. It is difficult to estimate this error, but experience suggests that samples with a plane surface at the mass are not showing measurable deviations. The distance
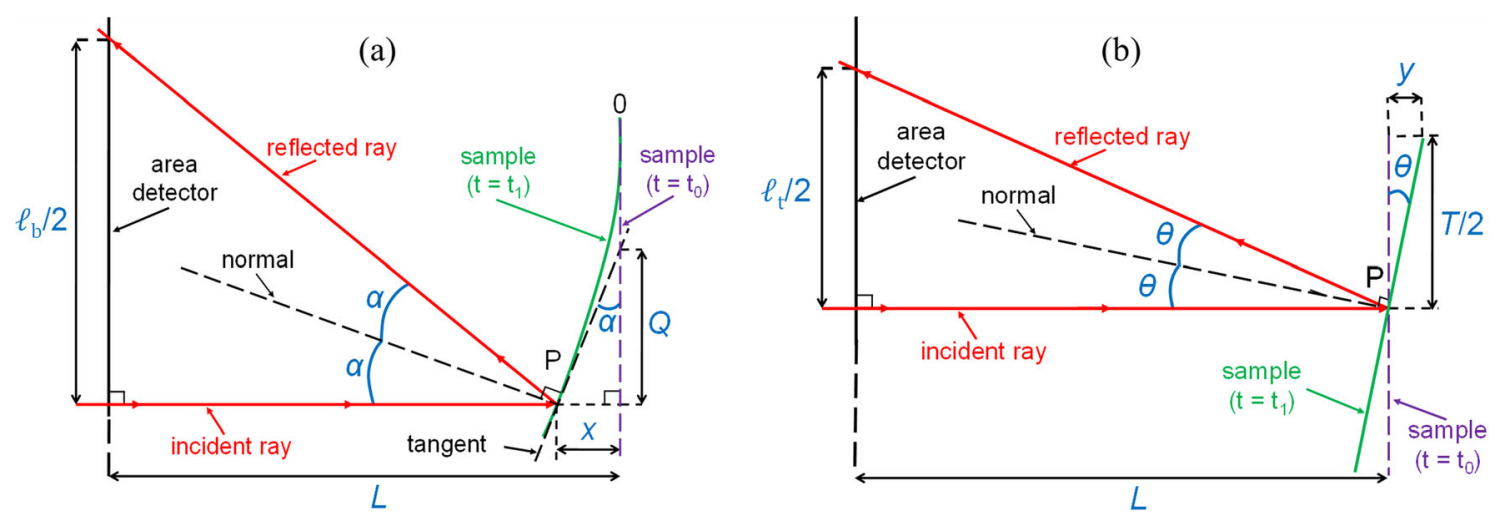

Fig. 5 Geometric calculation of the sample displacement $x$ for bending (a), and of the angle of rotation $\theta$ for torsion (b) 
between the sample and the area detector $L$ is also linearly positioned. This distance is controlled by a micro screw with a resolution of $10 \mu \mathrm{m}$ and corresponds to a $0.06 \%$ error. Another error is introduced by the area detector, which can detect the reflected beam position with a resolution of $1 \mu \mathrm{m}$, corresponding to a $0.03 \%$ for a typical VHCF test. Also, if the surface roughness of the sample is too high, the reflected beam is broadened and parts of the reflected beam could lie outside the sensor area. This error can be prevented by limiting the sensor range and optimizing the optical path so that the reflected laser beam stays on the area detector. The displacement sensor in the piezo actuator has a nominal resolution of $1.2 \mathrm{~nm}$ for their $60 \mu \mathrm{m}$ displacement range $(0.002 \%$ error $)$. This is the smallest displacement amplitude size the PID is able to control. To prevent timing issues, all signals are routed as analog signals and are digitized at the same time by the FPGA using the same reference clock. The FPGA clock is working at a frequency of $40 \mathrm{MHz}$. The time resolution is therefore determined by the FPGA and is in the range of $25 \mathrm{~ns}$. In theory there could be time lags for the different length of cables, but relative to the time resolution they are negligible.

\section{Experimental Implementation}

\section{Sample Preparation}

In this study, experiments have been performed with annealed nickel (99.00\%), and Oxygen-Free High Thermal Conductivity (OFHC) copper (99.95\%) samples acquired from thin metal foils (Goodfellow, UK). The hourglass shaped samples having a width of $500 \mu \mathrm{m}$ were cut from $\mathrm{Cu}$ thin foils with a thickness of $200 \mu \mathrm{m}$, while the Ni samples had a $1 \mathrm{~mm}$ long straight section with a $500 \times 200 \mu^{2}$ cross section. The Ni samples were shaped by a laser cutting process, while the $\mathrm{Cu}$ ones were cut by electrical discharge machining (EDM). In a consecutive process, all samples were electropolished in the gage section in order to remove thermally affected zones from manufacturing and to improve the surface quality in order to avoid the influence of surface defects on the damage formation. The surface roughness was measured with a 3D laser scanning microscope (VK-9700, Keyence, Japan). The roughness before electropolishing on both types of samples was $R_{a}=0.164 \mu \mathrm{m}$ and $R_{z}=3.125 \mu \mathrm{m}$, while after electropolishing and before cyclic loading, it was $R_{a}=0.068 \mu \mathrm{m}$ and $\mathrm{R}_{\mathrm{z}}=$ $1.844 \mu \mathrm{m}$. Manufacturing imperfections resulted in variations in dimensions; thus, each sample was measured individually.

The torsional and bending peaks of a measured frequency sweep are represented in Fig. 6. The bending and torsional amplitudes have maxima at $286 \mathrm{~Hz}$ and $293.5 \mathrm{~Hz}$ respectively. It should be noted that the frequencies of the two peaks are lower than the resonant frequencies calculated by FEA. This

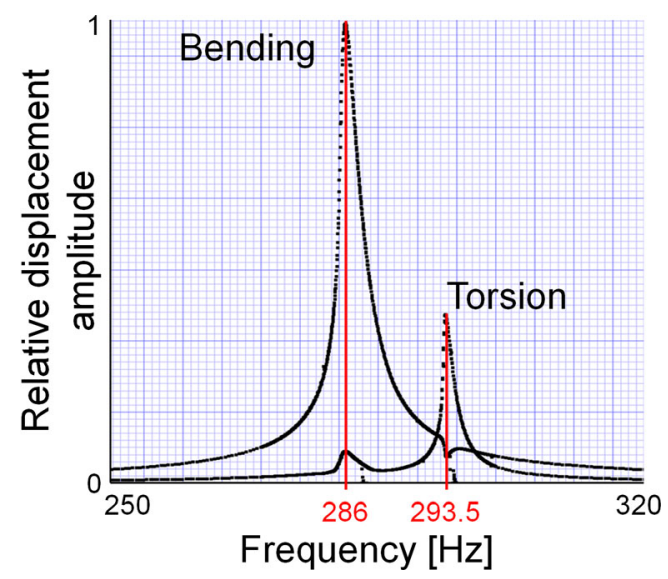

Fig. 6 Screen shot from the setup showing the measured displacement amplitude of a Ni sample for a frequency sweep with two peaks, one for bending and one for torsion

difference can be explained by geometric imperfections due to the fabrication and preparation of the samples, as well as by damping (e.g., due to air drag), which is not included in the linear elastic FE model. The reduction of the gage section dimensions, during manufacturing or electropolishing, was identified as the most contributing factor due to the reduction in stiffness.

Variations in manufacturing make it necessary to correct the mass distribution before the test to close the frequency gap between torsion and bending. Therefore, FE simulations were performed to find an optimally designed mass distribution in form of small added masses. Adding one drop of glue at the center of the mass leads to a decrease of the resonant bending frequency. If the drop is not placed at the center but shifted to one side of the mass, a mixture of a bending and torsional mode of the sample is observed. In case of two symmetrically applied drops, the resonant frequencies of both modes are affected. With increasing distance between the two drops from the center of the mass, the resonant bending frequency stays constant while the torsional one is reduced. A critical distance can be found, for which the two frequencies are equal. Finding this critical distance by simulation and recreating it in an experimental setup are completely different matters. In an attempt to bring the torsion and bending modes to the same frequency, super glue and lead balls were added with the largest distance from the center of the mass, at the end of the mass wings. Most trials resulted in a reduction of both resonant frequencies, and also tended to bring the modes much closer, but it is very difficult to apply the masses with the accuracy required to attain matching torsion and bending resonant frequencies. Although it is very difficult to attain matched modes, the addition of masses does noticeably increase the measured torsional amplitudes, allowing for an almost perfect multiaxial condition. Finally, as a quantified example, placing a $5 \mathrm{mg}$ lead ball at the middle of the mass leads to a decrease of $12 \mathrm{~Hz}$ of the bending resonant frequency. 
Using a precision balance with a $0.1 \mathrm{mg}$ resolution allows a controlled minimum resonant frequency change of $0.24 \mathrm{~Hz}$. Adding more weight to the mass does not change the mass distribution in the sample measurably. Furthermore, as the overall geometry stays the same, the stress distribution in the gage section is not influenced. This was also evaluated by FE simulations.

\section{Fatigue Testing Procedure}

Since the sample is driven at resonance, the control of the amplitude becomes a critical issue when fatigue damage occurs. Therefore, a precise control of the amplitude is of absolute necessity to the quality and reliability of the experiments. Due to the system slow response time at resonance, the control was performed only every $500 \mathrm{~ms}$ by a PID controller, a type of closed-loop feedback regulation. For this particular fatigue setup, two output signals are regulated: the bending and torsional amplitudes. The standard governing equation for PID controllers follows: $u(t)=K_{p}\left[e(t)+\frac{1}{T_{i}} \int_{0}^{t} e(\tau) d \tau+T_{d} \frac{d}{d t} e(t)\right]$. Where $u(t)$ is the modified variable output, $e(t)$ is the error between desired signal and measured signal, $K_{p}$ is the proportional gain, $T_{i}$ is the integral time, and $T_{d}$ is the derivative time. A systematic approach to finding these PID constants was desired to increase process reliability. A PID control with quarter wave decay was achieved through the application of the Ziegler-Nichols method, and the Tyreus-Luyben tuning coefficients were used to enhance robustness and decrease output oscillation.

The Ziegler-Nichols method requires the user to find two values, namely, the critical gain and oscillatory period at this critical gain. To clarify, the critical gain is the value of the proportional coefficient at which the output neither converges nor diverges; rather, it remains in oscillation. At this critical gain, one must measure the period of oscillation in order to apply the Ziegler-Nichols heuristic values. With these two values noted, one can also apply the Tyreus-Luyben state points. While the Ziegler-Nichols and Tyreus-Luybens methods provide an excellent starting point for understanding the effects of a PID controller on our custom built system, there is no 'one size fits all' blanket method of ensuring a well-controlled process. Neither the Ziegler-Nichols nor the Tyreus-Luyben values resulted in a stable stress state. For this reason, it was necessary to further examine the results that these methods produced, and attempt to reconcile them with fatigue experimentation in a non-perfect environment. To this end, one must understand how each constant, P, I, or D, affects the process being controlled. An explanation of the behavioral characteristics of $\mathrm{P}, \mathrm{I}$, and $\mathrm{D}$ is required for further discussion.

For processes with frequent large amplitude disturbances, literature suggests that the proportional P gain should dominate the PID controller response output [22]. Using a higher integral I gain results in a system that 'remembers' past errors, and is suitable in systems with long-lasting, low-amplitude perturbations. The $\mathrm{D}$ gain utilizes the slope of the present error to estimate future behavior of the system, and is useful in slowing very sudden and sharp changes in system output. Because the designed system in this situation is a very high cycle fatigue setup potentially working at its resonant frequency, an assumption is made that there will be low amplitude, long-duration perturbations, and perhaps every once in a while a sharp error stemming from some critical microstructural change. With this intuition taken into consideration, a controller with plant corrections dominated by the I and $\mathrm{D}$ gains is a logical choice. The $\mathrm{P}$ gain should not be emphasized in this case, as the typical perturbations will not be uniform across the entire time spectrum and also will not be high in amplitude or frequent in occurrence. Table 1 shows the variation between the $\mathrm{ZN}$, TL, and chosen constants for the fatigue setup that were found by empirical results and the above-stated logic. The above written standard form of PID equations was used in this discussion, however, the numerical implementation in LabView is slightly different due to its discretization method.

It should be noted that the most important difference between the proposed values is their reliance on proportional changes. A valid fatigue test simply cannot allow the large changes in the stress amplitude that would occur from a proportionally dominated control system. In order to decrease the varying effects of user error on each different experimental run, the empirically discovered PID gains could be programmed into LabView along with the heuristic base of the $\mathrm{ZN}$ and TL methods. This would allow the user of the test setup to consistently find proper $\mathrm{P}$, I, and $\mathrm{D}$ values for a wide range of samples $(\mathrm{Cu}$, or Ni with varying masses added) without fear of altering previously consistent test conditions.

Since the setup is intended for fatigue tests at resonance, it is clear that the stress amplitude must be tightly controlled to ensure test validity. What is not so directly obvious is that the input oscillation frequency must also be controlled due to the effect that fatigue damage has on stiffness over time. Stiffness is correlated to resonant frequency, and this is tracked in the setup by the phase angle between the actuator input and the sample response oscillation. A heavily customized proportional differential controller with adjustable slope and accuracy settings was programmed into LabView to control the resonant frequency. The slope of the frequency controller can be adjusted by the user to incorporate actual sweep data in the

Table 1 PID values from their respective tuning method

\begin{tabular}{llll}
\hline & $\mathrm{P}\left(K_{p}\right)$ & $\mathrm{I}\left(T_{i}, \mathrm{~min}\right)$ & $\mathrm{D}\left(T_{d}, \mathrm{~min}\right)$ \\
\hline Ziegler-Nichols & 0.307 & 0.0595 & 0.0149 \\
Tyreus-Luyben & 0.237 & 0.269 & 0.0189 \\
Derived empirical values & 0.0270 & 0.396 & 0.0287 \\
\hline
\end{tabular}


control regime. When the phase is controlled close to $90^{\circ}$, it means that the sample is being actuated at either its torsional or bending resonant frequency, and as stated, deviation from one of these modes at resonance can drastically affect the stress amplitude. Because of this behavior, the measured output frequency must be controlled to maintain resonance. While normal usage of the frequency controller is intended for a phase of $90^{\circ}$ exactly at one of the modes, it is also often desirable to control out of mode, when strong multiaxial states are present in the sample. To give the user the option to move away from perfect modal control, a variable target phase was programmed as a function of the setup.

In this setup, the testing methodology is optimized by using the aforementioned two controllers. The resonant frequency is first defined by sweeping the driven frequency. When a rough value is found, the proportional controller for the resonant frequency is started. The driven frequency is stabilized at the sample resonant frequency by controlling the phase angle at $\Phi=90^{\circ}$. The amplitude of the sample is then slowly increased to reach the desired testing amplitude. It is important that the amplitude gradually rises. Since the resonant frequency and the amplitude are strongly correlated, an amplitude increase results in a frequency shift, which the resonant frequency proportional controller must be able to compensate for. When the testing amplitude is reached, the amplitude PID controller is started. Both controllers then work in parallel to ensure a constant resonant state and a constant amplitude. After this point, the test is in its optimized conditions and damage formation can be tracked.

It is known that the frequency, $f$, is proportional to the square root of the stiffness, $k$, over the mass, $m$. A reasonable assumption is made that the mass stays constant during every test. The next assumption is, that the stiffness of the sample decreases with damage formation. With the setup at hand it is possible to measure a small decrease in the resonant frequency, and thus indirectly measure the appearance of defects. A frequency sweep is done before each test (See Fig. 7). Taking as an example a bending test of a Ni sample, a change of $\Delta \Phi=1^{\circ}$ of the phase angle (between the piezo actuation and the sample oscillation) around the resonant frequency corresponds to a relative frequency change of $\Delta f / f_{\text {initial }}=5 \times 10^{-5}$. The measured phase angle showed a noise floor of roughly $\Delta \Phi=0.01^{\circ}$. Thus the resolution of the relative resonant frequency is about $\Delta f f f_{\text {initial }}=5 \times 10^{-7}$.

\section{Results}

Bending experiments on $\mathrm{Ni}$ and $\mathrm{Cu}$ micro samples were conducted in the HCF regime to validate the experimental resolution of the setup. Due to the accumulating fatigue damage, the resonant frequency of the bending micro samples decreases with increasing cycle number. In Fig. 8, the typical relative resonant frequency change $\Delta f / f_{\text {initial }}$ versus cycle number of a micro sample is shown. Up to $N=10^{5}$ cycles, the resonant frequency is only slightly changing by roughly $\Delta f f f_{\text {initial }}=1 \times 10^{-4}$. In this regime, extrusions develop in many grains and consecutively micro cracks initiate in individual grains. Beyond $10^{5}$ cycles the relative frequency change decreases below $\Delta f f f_{\text {initial }}=1 \times 10^{-3}$ and micro cracks propagate through grain boundaries to form larger cracks. After a relative frequency decrease beyond roughly $\Delta f f f_{\text {initial }}=5 \times 10^{-3}$ a large crack propagates through the sample changing the resonant frequency at a fast rate.

The first slip bands were accompanied by a decrease of the relative resonant frequency on the order of roughly $\Delta f f f_{\text {initial }}=10^{-4}$ (Fig. 9). At this stage, focused ion beam (FIB) cross sections showed no observable cracks. Figure 9(a) is a laser scanning micrograph of the sample surface, where each dark line represents an extrusion. The laser scanning microscopy allows quantitatively tracking the extrusion height formation but is limited in spatial resolution. Figure 9(b) is an ion-enhanced electron micrograph, it shows the grain morphology of the sample surface with an intense grain orientation contrast. Each dark line (highest ones indicated by white arrows) represents an extrusion constrained within the grain boundaries. At this stage, the extrusions are typically constrained to individual grains, though several similarly-oriented neighboring grains show extrusions crossing their grain boundaries. Micro cracks could not be found at this stage of fatigue damage accumulation in the samples under investigation.

In the next stage, the extrusions and intrusions are increased in their amplitude, leading to crack initiation. In this case a $\mathrm{Cu}$ micro sample showed a resonant frequency decrease of $\Delta f f f_{\text {initial }}=1 \times 10^{-3}$ corresponding to multiple crack initiations at the surface. Figure 10(a) shows a scanning electron micrograph (SEM) of a fatigued $\mathrm{Cu}$ grain. Only ten such grains are fatigued on the upper sample surface. The red line represents where the sample has been cut as seen in Fig. 10(b). The focused ion beam cross section shows the initiated defects growing from the surface and penetrating into the depth. After a relative decrease of $\Delta f f f_{\text {initial }}=10^{-3}$ the fatigue induced surface roughening has increased dramatically, as can be seen in Fig. 11(b) for a Ni micro sample. Figure 11(a) illustrates the 'as prepared' sample and its electropolished surface. Figure 11(b) shows the sample after $N=10^{6}$ cycles under a bending stress amplitude of $\sigma_{\mathrm{a}}=200 \mathrm{MPa}$. Both images were acquired with a laser scanning microscope. Extrusions and cracks appeared at the surface, where the maximum stress amplitude is expected from FE simulations (Fig. 11(c)). At this stage, several micro cracks can be observed at the sample surface. Figure 11(d) is a magnification of Fig. 11(b) and shows the damage in the straight section. A further 
Fig. 7 Experimental sweep of the amplitude and phase angle for a micro sample under a bending load

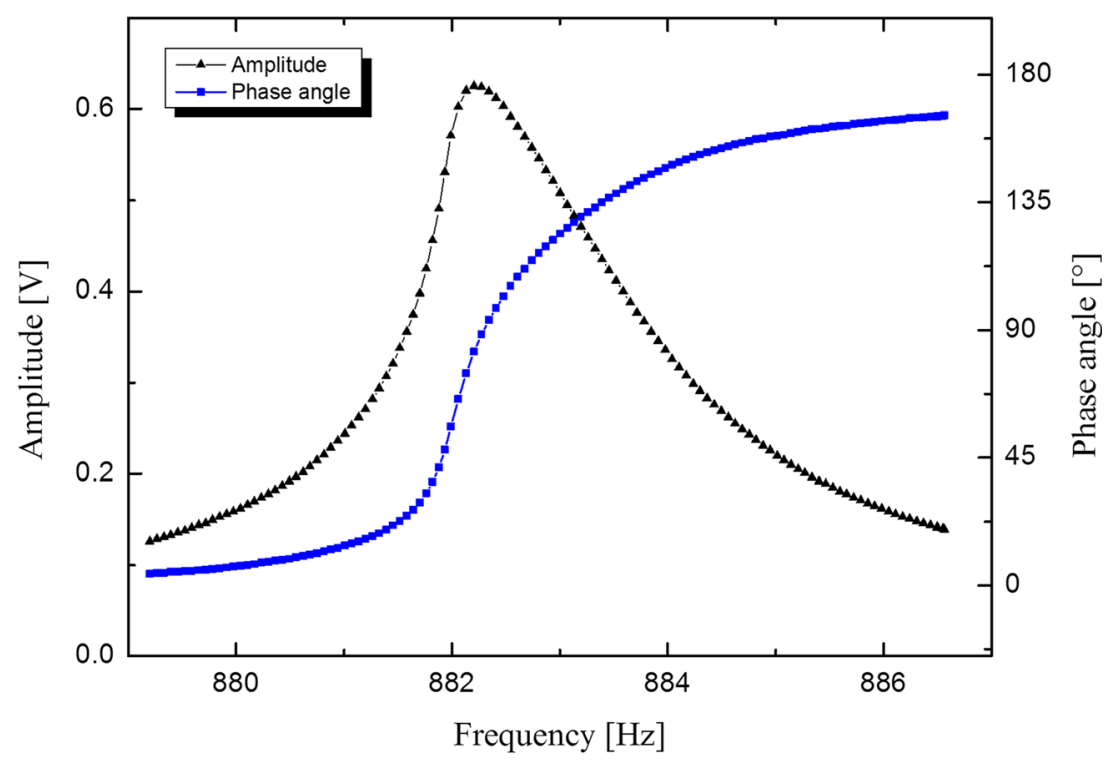

magnification (Fig. 11(e)) shows that the extrusions (white lines) are covered by Ni oxide, while the crack (dark line) follows a path along extrusions and crosses a grain boundary into the neighboring grain (see arrows). The orientation of the crack is changing at the grain boundary. Yang [23] and Boyce [24] report similar stress corrosion cracking for Ni samples.

Finally, at a relative resonant frequency decrease of $\Delta f f f_{\text {initial }}=$ $10^{-2}$ one of the micro cracks propagates through the sample. Figure 12(a) shows multiple cracks at the sample surface going through the width and the thickness. The FIB cross section in Fig. 12(b) documents how the crack propagates through the grains and crosses grain boundaries. At this stage, the sample is considered to have failed. It is not possible anymore to track the phase angle between the actuation and the sample response. The resonant mode has become unstable.

\section{Discussion}

\section{Comparison of Crack Initiation Detection Methods}

The implementation of the presented custom built resonant multiaxial micro fatigue setup allows for investigations of fatigue at a variety of size scales and loading scenarios. The setup has a very fine measurement resolution of the relative resonant frequency change $\Delta f f f_{\text {initial }}=5 \times 10^{-7}$. The strong correlation between the resonant frequency change and the damage evolution indicates that the increasing plasticity, resulting in extrusions, intrusions, crack initiation, and crack growth, can lead to a stiffness decrease of the sample and therefore directly impacts the resonant frequency. Since the experiments are strain controlled, the impact of plasticity on resonant
Fig. 8 Typical relative resonant frequency shift versus cycle number compared to defects in a Ni sample

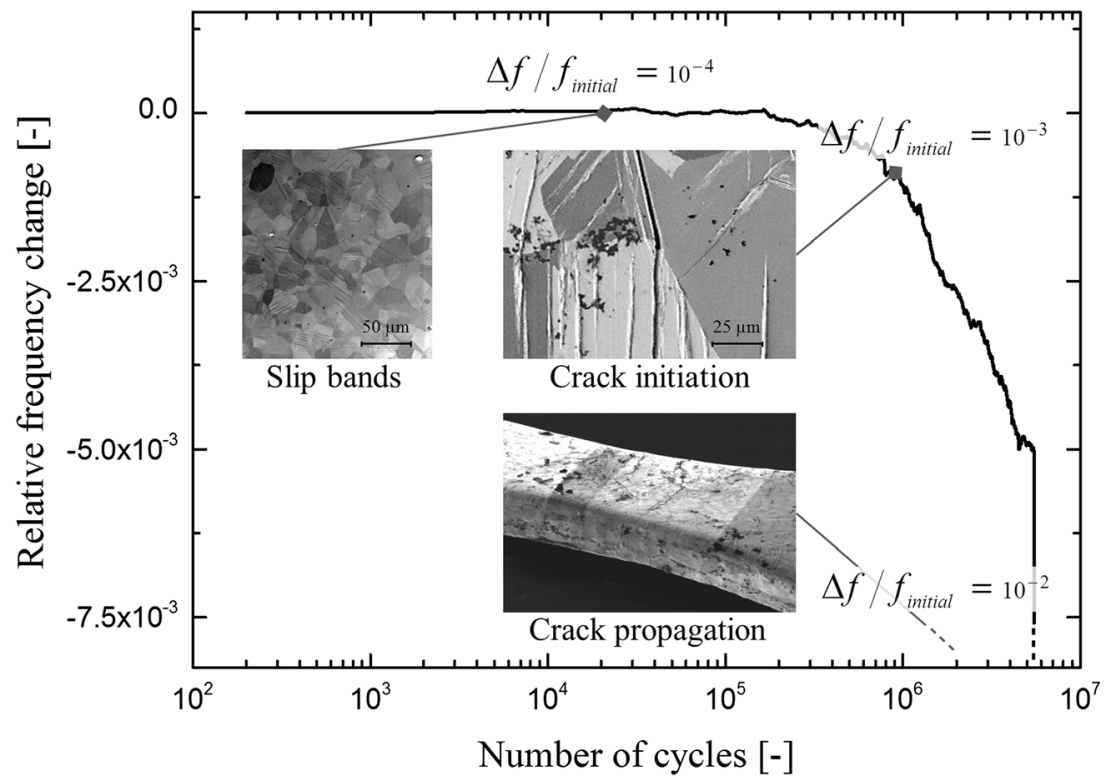


Fig. 9 Laser scanning micrograph (a) and an ionenhanced electron micrograph (b) of slip band formation at the same location after a relative resonant frequency decrease of $\Delta f / f_{\text {initial }}=$ $10^{-4}$ on a Ni sample. The white arrows point out the highest persistent slip bands

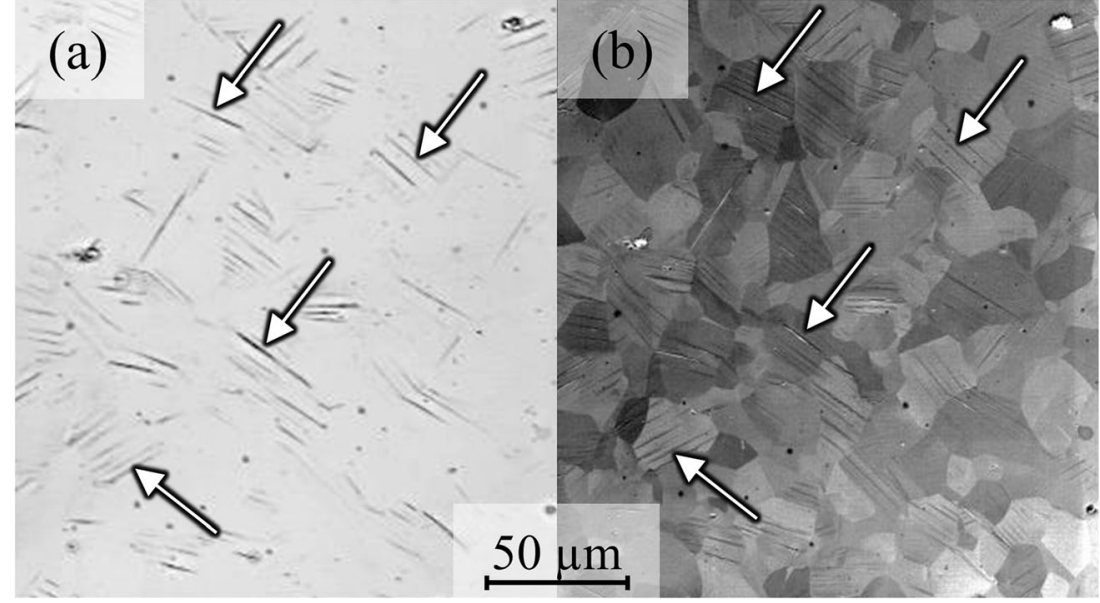

frequency can be explained by a cyclic softening. This softening leads to a decrease in the stress amplitude and hence the slope of the stress-strain hysteresis is reduced. This decrease could be compensated, as it is in the work of Yang et al., by changing the position of the piezo actuator to maintain mean load $[25,26]$. Nevertheless this is not the case in this manuscript. With the initiation of cracks, the stiffness of the sample is even further decreased. While the elastic effect of micro cracks in a large sample cannot be picked up in the mechanical response due to the intrinsic and extrinsic damping, micro cracks in a small sample volume will have a much higher signal to noise ratio and can be tracked. Here scaling effects are beneficial as the intrinsic damping scales with volume, and therefore is reduced with sample size in a cubic fashion while the crack initiation will only take place at the surface and therefore only scales in a quadratic fashion. With increasing number and length of cracks, the resultant impact on stiffness becomes more and more apparent.

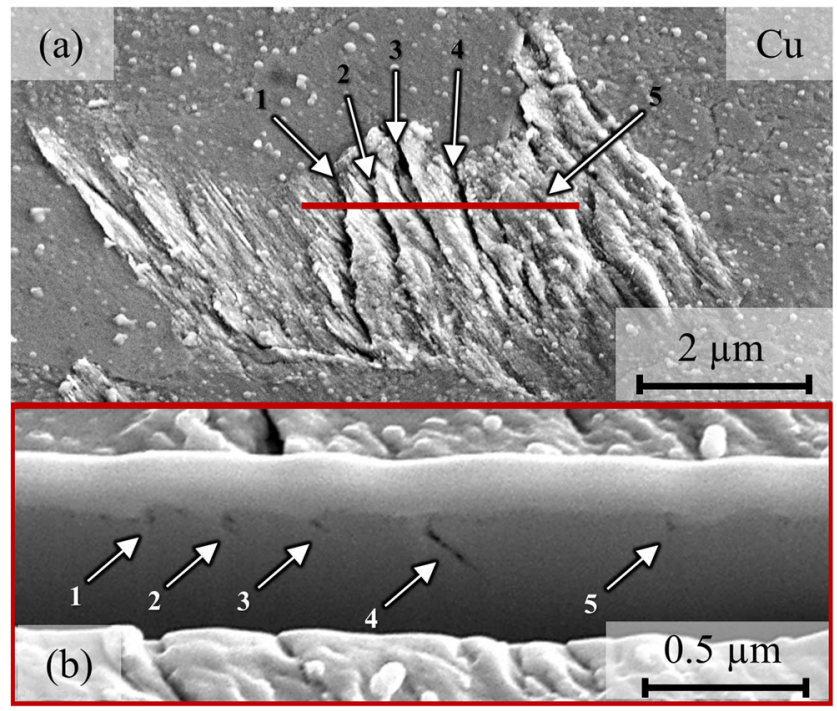

Fig. $10 \mathrm{Cu}$ sample: (a) Scanning electron micrograph (SEM) of a fatigued grain after a relative resonant frequency decrease of $\Delta f f f_{\text {initial }}=10^{-3},(\mathbf{b})$ SEM of a FIB cross section: micro crack formation at extrusions
This allows the accurate tracking of fatigue damage formation prior to crack initiation such as plasticity induced surface roughening and slip band formation (Fig. 9) at a state where it appears in only a few grains. The orientation of these grains can be found performing Electron Back Scatter Diffraction (EBSD) imaging, providing information about the crystal lattice planes where damage accumulation initiates and later

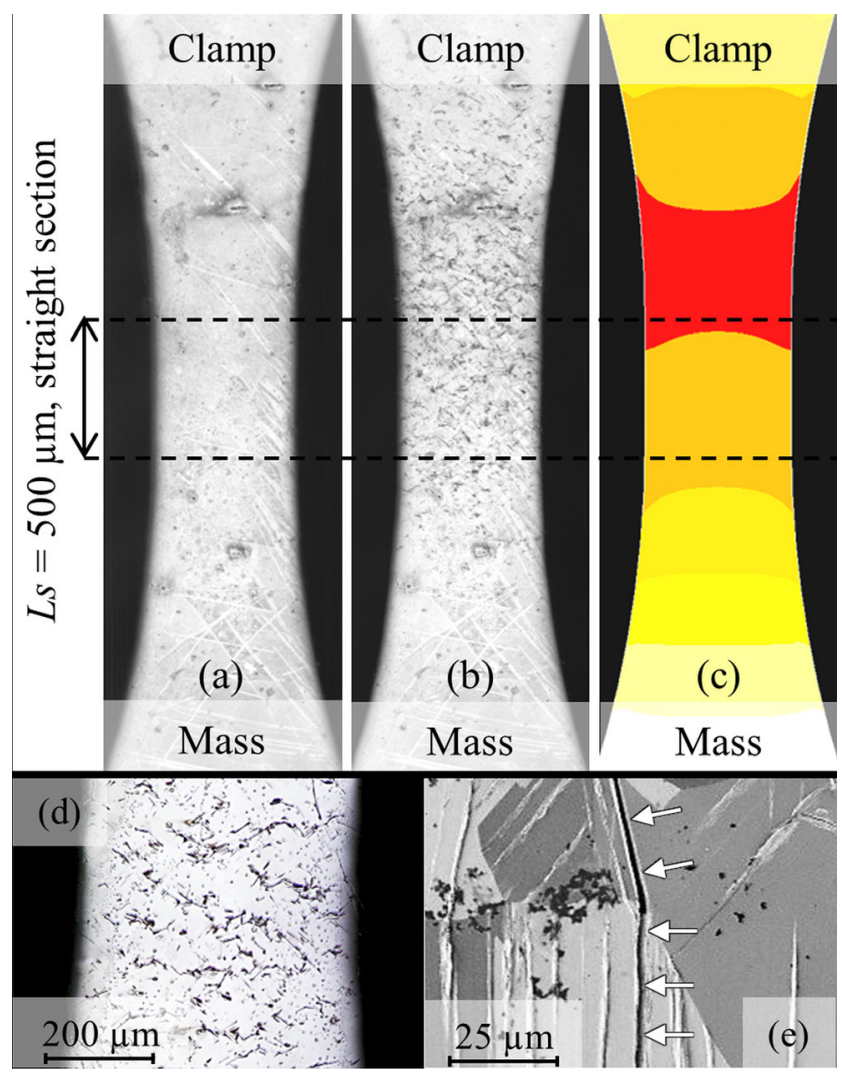

Fig. 11 Laser scanning micrographs: (a) Ni sample before fatigue, (b) after $N=10^{6}$ cycles at $\sigma_{\mathrm{a}}=200 \mathrm{MPa}$ stress amplitude. (c) Stress distribution according to FE simulation. (d) Laser scanning micrograph, (e) scanning electron micrograph of the crack initiation after a relative resonant frequency decrease of $\Delta f f f_{\text {initial }}=10^{-3}$ 
Fig. 12 (a) Scanning electron micrograph, (b) SEM of a FIB cross section showing the crack propagation after a relative resonant frequency decrease of $\Delta f f f_{\text {initial }}=10^{-2}$ on a Ni sample

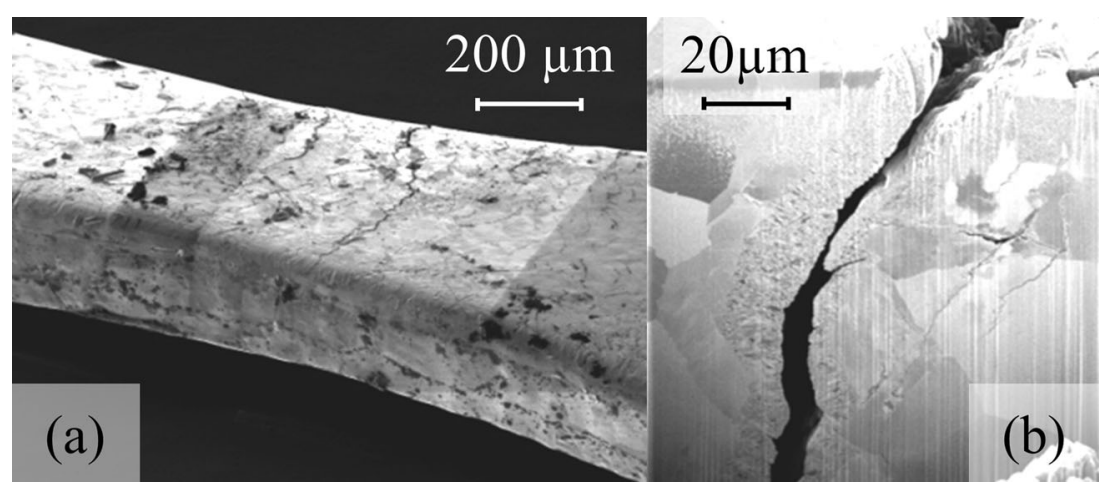

leads to fatigue failure. This setup is a novel way of experimentally examining crack initiation processes. Crack initiation and the subsequent short crack formation and growth play a particularly important role in the $\mathrm{HCF}$ and VHCF regimes, they very strongly determine the material lifetime. Therefore, this method allows the examination of the microstructural features that lead to fatal failure, and could thus advance the understanding of how cracks nucleate during fatigue. The high sensitivity allows the definition of experimental 'stop cycling' criteria, through which one can observe damaged structures right after the appearance of extrusions, micro cracks (smaller than 500 nm, Fig. 10(b)), and the crack propagation behavior. Although the setup is best suited for the detection of surface damage, it is also capable of detecting damage in the interior volume by tracking the frequency change. This internal damage onset would then be relatively difficult to locate (FIB and SEM required), when compared to surface damage, but it is possible.

Other techniques to track crack initiation and propagation are e.g., the replica technique, electrical damage detection, thermal methods, and the analysis of the higher harmonics in sample behavior. The replica technique is a well-known method for examining the crack profile of macro samples [27]. After being moistened in acetone, a cellulose acetate sheet is pressed against the sample surface. The replica is then peeled off, coated e.g., by $\mathrm{Au}$, and then examined in an SEM. This method allows post-mortem analyses at different fatigue stages while the sample remains in the testing machine. However this method does not allow the continuous tracking of crack formation and growth. Furthermore, it is necessary to apply a load to imprint the surface roughness and is thus not well suited to be used on fragile micro samples. Another way of tracking crack initiation and propagation is electrical damage detection. The electrical resistance of a sample depends on its cross section, which becomes smaller as cracks propagate. Early electrical resistance changes can be attributed to the initiation of voids, dislocations and micro cracks. However, it is not always possible to identify micro cracks deeper than $0.1 \mathrm{~mm}$ [28]. Sun et al. showed that fatigue lifetime prediction and failure [29], can be established, but the detection of crack initiation remains difficult. For an experiment operating at the resonant frequency of the sample, it is difficult to attach electrical connections without influencing the vibrational mode. This can be circumvented by infrared thermography methods [30]. The temperature field on the sample surface is observed by an infrared camera. At the crack initiation site, thermal dissipation leads to a surface temperature increase. That said, the spatial resolution of the infrared camera limits the detection of micro crack formation. These two methods, infrared thermography and electrical resistance measurement, could also be combined to determine a new lifetime calculation [31]. Another mechanical method, which can be implemented as a non-contact in-situ measurement of non-linear behavior was introduced by Kumar et al. and Funk et al. [32, 33]. The authors describe their method as a nonlinear ultrasonic measurement via analysis of the feedback signal of a closed-loop ultrasonic fatigue system, where the amplitude of the higher harmonics of the feedback signal is analyzed. The authors argue that the current damage state leads to a specific nonlinear behavior, corresponding to changes in the amplitude of the higher harmonics of the (applied) fatigue frequency. Both internal and surface-initiated cracks, as well as initial hardening/softening, and fatigue crack growth can be detected. So far, the characteristic answer is difficult to trace back to a specific damage mechanism, although this might be possible in the future.

In conclusion, the new method described in this paper generates additional insights on crack initiation. The term crack initiation should nevertheless be clarified since it is directly correlated to the different crack observation methods. Zimmermann [34] concluded in her review that the nucleation of a fatigue crack could be described as the damage evolution from the atomic scale up to $1 \mu \mathrm{m}$. It is followed by micro cracks and short cracks, with a size between $1 \mu \mathrm{m}$ and $1 \mathrm{~mm}$, and with everything above being considered a long crack. Other experimental setups have recently been developed to look at the early damage initiation; for example, in medium carbon steel considering generator power and specimen temperature [35] or in carbon fiber reinforced plastics using fast Fourier, short time Fourier, and Hilbert Huang 
transformation [36]. Nevertheless, none of them can observe a relative resonant frequency change corresponding to a crack length smaller than $1 \mu \mathrm{m}$. In contrast, smaller samples allow for a higher signal to noise ratio concerning the crack initiation, as the initiated cracks contribute much more to the sample behavior than in a macroscopic sample.

\section{Experimental Investigation on OFHC Cu}

The results from the experimental investigation on OxygenFree High Thermal Conductivity (OFHC) $\mathrm{Cu}$ micro bending samples are compared to bulk material samples and thin films in Fig. 13. It shows that dimensional constraints cause size effects in small-scale samples. It has to be noted though, that the comparison between the experimental lifetime results should be reviewed critically due to the different lifetime criteria.

Considering the macro scale, OFHC Copper cylindrical specimens of $2.5 \mathrm{~cm}$ gauge length and $0.8 \mathrm{~cm}$ diameter with a grain size between 3.4 and $150 \mu \mathrm{m}$ were fatigued (fully reversed loading at $30 \mathrm{~Hz}$ ) by Thompson and Backofen [37]. OFHC Cu (99.95\%) with a grain size of $30 \mu \mathrm{m}$ was also studied by Phung et al. [14] with fully reversed loading at $20 \mathrm{kHz}$. Phung et al. used cylindrical and flat samples having gauge lengths of about $3 \mathrm{~cm}$, with the cylindrical samples having diameters of $3 \mathrm{~mm}$, and the flat samples a cross section of $3 \times 2 \mathrm{~mm}$. The difference between the lifetime measured by Thompson and Backofen and Phung et al. could be due to the preparation of the OFHC $\mathrm{Cu}$, or other experimental differences, a notable difference being the extremely different test frequencies $(30 \mathrm{~Hz}$ for Thompson and Backofen, $20 \mathrm{kHz}$ for Phung et al.). In contrast, the micro bending fatigue tests from this study use a failure criterion related to the decrease of the relative resonant frequency $\left(\Delta f / f_{\text {initial }}=1 \times 10^{-3}\right)$, which was associated with crack initiation. It can be argued that in the

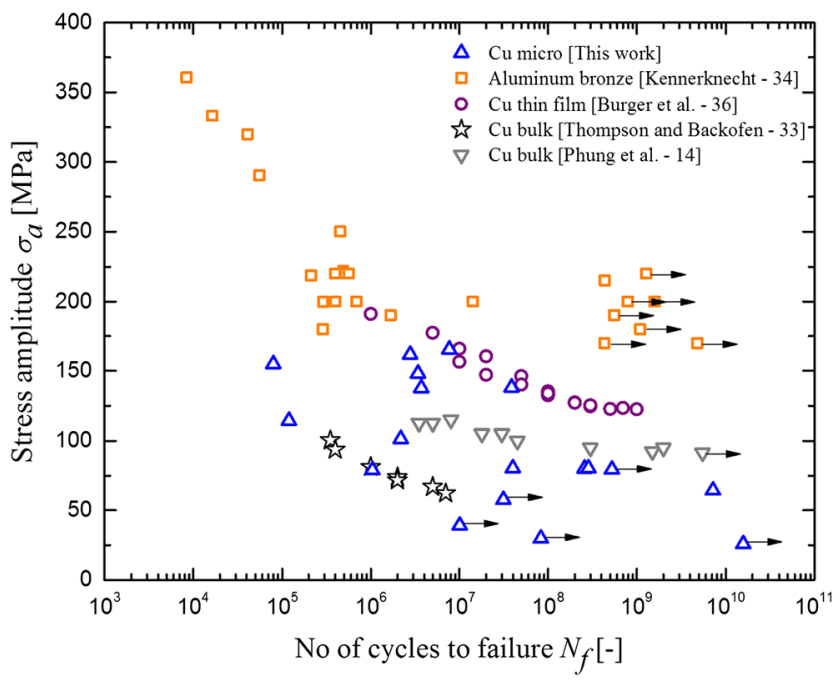

Fig. 13 OFHC Cu lifetime diagram higher HCF and the VHCF regime, the practical lifetime of micro samples is mainly determined by crack initiation. Therefore, the two lifetime criteria will lead to similar fatigue lifetimes in that regime.

The fatigue lifetime of $500 \times 200 \mu \mathrm{m}^{2} \mathrm{OFHC} \mathrm{Cu}$ specimens measured with the custom built resonant multiaxial micro fatigue setup show altogether a higher lifetime compared to values from Thompson and Backofen for a comparable grain size. Although the lifetime data shows significant scatter, the lower lifetime limit is the same as for macroscopic samples. Thus, such specimens are large enough to carry a representative distribution of defects or microstructural features. Small samples, such as most of the micro OFHC Cu specimens, are therefore not representative elements of the bulk material, and show a widely-distributed lifetime. As a consequence, micro samples have on average a higher lifetime, but carry a high risk to fail, as their lifetime distribution is wider than that of larger samples. Comparing these results, it can be concluded that the lifetime of the $\mathrm{Cu}$ micro samples is controlled by a statistical size effect. For the bending fatigue tests of $\mathrm{Cu}$ micro samples, the highest stressed volume is limited to only a couple hundred grains. Hence, it can be argued that these samples are not representative elements of the natural defect distribution. Nevertheless, such non-representative distributions are often used in applications such as micro components cut out of a macro material. Such samples are also useful for testing a targeted micro structure and investigating specific fatigue mechanisms. Finally in terms of the $\mathrm{Cu}$ micro samples, the probability is low that arrangements of grains are present which can lead to damage accumulation crossing several grains, resulting in fatal cracks [15]. This is similar to the findings of Furuya [19] who has shown that the sample size affects the lifetime of high strength steel in the VHCF regime. Furuya's results show that the scatter of the sample lifetime increases with decreasing sample size, but only after the samples become smaller than a certain critical volume.

The initial defect distribution is typically related to the manufacturing or deposition process, and the subsequent thermal treatment. The sample lifetime can be influenced by changing the manufacturing parameters. This is well illustrated by the uniaxial fatigue tests with micro molded tensile samples (cross section $260 \times 130 \mu \mathrm{m}^{2}$, gage length $1 \mathrm{~mm}$ ) made of aluminum bronze (CuAl10Ni5Fe4) [38]. The lifetime of the CuAl10Ni5Fe4 micro samples in contrast shows a limited scatter. This seems inconsistent with Furuya's argument, but the reason for this behavior can be found in the very fine microstructure resulting from the manufacturing process. While the $\mathrm{Cu}$ micro samples were extracted from a large $\mathrm{Cu}$ foil, the $\mathrm{CuAl10Ni5Fe} 4$ samples were micro molded directly into the final shape [39]. Therefore, the samples contain all defects and microstructural features developed during the molding process and are representative elements for the characteristic defect distribution. 
These results can also be compared with thin film fatigue tests to further explore the size effects. $1 \mu \mathrm{m} \mathrm{Cu}$ thin films were sputter deposited on the Si cantilevers with dimensions of $10 \times 1 \times 0.2 \mathrm{~mm}^{3}$, and the cantilevers were then subjected to piezo actuation [40]. The failure criterion for the thin film samples needed to be chosen differently since the thin film is attached to the substrate. Because the Si substrate in this setup is two orders of magnitude thicker than the $\mathrm{Cu}$ microfilm deposited on top of it, as well as the fact that the substrate has a higher Young's modulus and yield stress, only the $\mathrm{Cu}$ microfilm is subjected to a significant fatigue stress amplitude. Therefore, a crack in the $\mathrm{Cu}$ thin film does not lead to a failure of the whole sample. During fatigue, extrusions form and increase the roughness of the thin film surface, which can be detected by the surface reflectivity. The surface reflectivity was thus chosen as the fatigue failure criterion. The thin film lifetime data does not show a lot of scatter and follows a constant decrease in lifetime. This can be explained by the large number of grains involved due to the small grain size of less than $1 \mu \mathrm{m}$ in relation to the large sample size $(1 \mathrm{~mm}$ wide cantilever) and the statistical grain size distribution.

Finally, the method described in this paper focuses on the crack initiation and short crack growth in the early stages, and cannot be used to observe the evolution of long cracks due to the sample size and applied stress gradients. While the stress gradient at the sample surface across the $500 \mu \mathrm{m}$ width is smaller than $2 \%$, the stress gradient through the thickness is much higher and therefore can interfere with cracks longer than $20-30 \mu \mathrm{m}$. At this length, the cracks can reach far enough into the sample depth that the stress at the crack tip is reduced. This is not true for cracks which reach through the full width of the sample. In this case, the neutral axis is shifted as well, and the stress intensity at the crack tip does not change. Nevertheless, relative to the sample size, a $20-30 \mu \mathrm{m}$ long crack is a mechanically rather severe crack, and the sample lifetime from this point on is rather short. In conclusion, even though the stress gradient in our micro samples could have an impact on crack evolution in the later stages, it is not the case for the lifetime during crack initiation, which is a large part of the lifetime in the VHCF regime; however, in the lower HCF regime, the crack growth plays a considerable role and therefore the experiments presented here might underestimate the materials lifetime.

\section{Summary}

A multiaxial resonant fatigue setup was developed in order to evaluate fatigue behavior and crack initiation in micro samples under multiaxial loading in the $\mathrm{kHz}$ regime. Through finite element analysis, optimal sample geometries were examined and parameterized in order to achieve the desired multiaxial stress states. Two piezo actuators drive the sample output motion. The desired torsional and bending amplitudes were controlled by a PID controller, and the excited frequencies were similarly regulated, resulting in stable experiments capable of achieving $10^{9}$ cycles within a reasonable experimental time frame. Real time sample monitoring is achieved through an area detector measuring the response of a laser beam reflected off of the micro sample surface, allowing a fine relative resonant frequency measurement resolution of $5 \times 10^{-7}$. Based on the results from three-stage damage formation in nickel samples, a material failure criterion related to the decrease in the micro sample relative resonant frequency was chosen and correlated to crack nucleation. Copper samples were tested to the very high cycle fatigue regime, and these results were compared to peer-reviewed experimental datasets. The resonant fatigue data show a comparable trend for micro samples in that they do not fail before bulk samples and have a relatively large scatter due to the lower likelihood of the presence of defect prone microstructural features. The multiaxial micro fatigue setup enables a unique in-situ experimental procedure that mixes high frequency testing capabilities with high resolution and reliable results, while also presenting a novel method of detecting and isolating fatigue damage from initiation to failure.

Acknowledgments The authors would like to acknowledge E. Ernst for his help at the institute, M. Meidlinger and J. Ye for their kind collaboration, and finally M. Buck for his critical review of our FE analysis. Furthermore, we acknowledge Prof. Kraft from the KIT for his support of the group. We would also like to acknowledge the funding by the German Science Foundation (Deutsche Forschungsgemeinschaft, DFG; EB362/41 SPP1466 and SFB499/3-2007 N01) as well as the KIT for financial support of our work. We would also like to acknowledge funding through the Fraunhofer Attract program.

Open Access This article is distributed under the terms of the Creative Commons Attribution 4.0 International License (http:// creativecommons.org/licenses/by/4.0/), which permits unrestricted use, distribution, and reproduction in any medium, provided you give appropriate credit to the original author(s) and the source, provide a link to the Creative Commons license, and indicate if changes were made.

\section{References}

1. Zhang G, Volkert C, Schwaiger R, Wellner P, Arzt E, Kraft O (2006) Length-scale-controlled fatigue mechanisms in thin copper films. Acta Mater 54(11):3127-3139

2. Straub T, Baumert EK, Eberl C, Pierron ON (2012) A method for probing the effects of conformal nanoscale coatings on fatigue crack initiation in electroplated Ni films. Thin Solid Films 526: 176-182

3. Eberl C, Courty D, Walcker A, Kraft O (2012) Stress-gradient induced fatigue at ultra high frequencies in sub-micron thin metal films. Int J Mater Sci 03, 1:80-86

4. Latham JI, Shreve WR, Tolar NJ, Ghate PB (1979) Improved metallization for surface acoustic wave devices. Thin Solid Films 64:915 
5. Eberl C, Spolenak R, Kraft O, Kubat F, Ruile W, Arzt E (2006) Damage analysis in Al thin films fatigued at ultrahigh frequencies. J Appl Phys 99:113501

6. Müller-Bollenhagen C, Zimmermann M, Christ HJ (2010) Very high cycle fatigue behaviour of austenitic stainless steel and the effect of strain-induced martensite. Int J Fatigue 32(6):936-942

7. Stanzl-Tschegg SE (2006) Proc fatigue crack growth and thresholds at ultrasonic frequencies. Int J Fatigue 28:1456-1464

8. Bathias C, Drouillac L, Francois P (2001) How and why the fatigue $\mathrm{S}-\mathrm{N}$ curve does not approach a horizontal asymptote. Int J Fatigue 23:143-151

9. Shiozawa K, Morii Y, Nishino S, Lu L (2006) Subsurface crack initiation and propagation mechanism in high-strength steel in a very high cycle fatigue regime. Int J Fatigue 28(11):1521-1532

10. Christ HJ, Mughrabi H (1996) Cyclic stress-strain response and microstructure under variable amplitude loading. Fatigue Fract Eng Mater Struct 19(2-3):335-348

11. Wang R, Mughrabi H, McGovern S, Rapp M (1984) Fatigue of copper single crystals in vacuum and in air: persistent slip bands and dislocation microstructures. Mater Sci Eng 65(2):219-233

12. Weidner A, Amberger D, Pyczak F, Schoenbauer B, StanzlTschegg SE, Mughrabi H (2010) Fatigue damage in copper polycrystals subjected to ultrahigh-cycle fatigue below the PSB threshold. Int J Fatigue 32, 6:872-878

13. Stanzl-Tschegg SE, Mughrabi H, Schoenbauer B (2007) Life time and cyclic slip of copper in the VHCF regime. Int J Fatigue 29(911):2050-2059

14. Phung NL, Favier V, Ranc N, Valès F, Mughrabi (2014) Very high cycle fatigue of copper: evolution, morphology and locations of surface slip markings. Int J Fatigue 63:68-77

15. Mughrabi H (2006) Specific features and mechanisms of fatigue in the ultrahigh-cycle regime. Int J Fatigue 28(11):1501-1508

16. Krupp U (2007) Fatigue crack propagation in metals and alloys: microstructural aspects and modelling concepts. Wiley

17. Stanzl-Tschegg SE (1999) Fracture mechanisms and fracture mechanics at ultrasonic frequencies. Fatigue Fract Eng Mater Struct 22, 7:567-579

18. Marines-Garcia I, Doucet JP, Bathias C (2007) Development of a new device to perform torsional ultrasonic fatigue testing. Int $\mathrm{J}$ Fatigue 29(9-11):2094-2101

19. Furuya Y (2008) Specimen size effects on gigacycle fatigue properties of high-strength steel under ultrasonic fatigue testing. Scr Mater 58(11):1014-1017

20. Schwaiger R, Kraft O (2003) Size effects in the fatigue behavior of thin Ag films. Acta Mater 51(1):195-206

21. Mattheck C (2006) Teacher tree: the evolution of notch shape optimization from complex to simple. Eng Fract Mech 73(12):17321742

22. Åström KJ, Hägglund T (2006) Advanced PID control. http://lup. lub.lu.se/record/535630
23. Yang Y, Imasogie BI, Allameh SM, Boyce BL, Lian K, Lou J, Soboyejo WO (2007) Mechanisms of fatigue in LIGA Ni MEMS thin films. Mater Sci Eng A 444:39-50

24. Boyce BL, Michael JR, Kotula PG (2004) Fatigue of metallic microdevices and the role of fatigue-induced surface oxides. Acta Mater 52:1609-1619

25. Yang Y, Allameh SM, Lou J, Imasogie BI, Boyce BL, Soboyejo WO (2007) Fatigue of LIGA Ni micro-electro-mechanical systems thin films. Metall Mater Trans A 38:2340-2348

26. Yang Y, Allameh SM, Boyce BL, Chan KS, Soboyejo WO (2007) An experimental study of fracture of LIGA Ni MEMS films. Metall Mater Trans A 38:1223-1230

27. Walker N, Beevers CJ (1978) A fatigue crack closure mechanism in titanium. Fatigue Eng Mater Struct 1(1):135-148

28. Charrier J, Roux R (1991) Evolution of damage fatigue by electrical measure on smooth cylindrical specimens. Nondestruct Test Eval 6(2):113-124

29. Sun B, Yang L, Guo Y (2007) A high-cycle fatigue accumulation model based on electrical resistance for structural steels. Fatigue Fract Eng Mater Struct 30(11):1052-1062

30. Wagner D, Ranc N, Bathias C, Paris PC (2009) Fatigue crack initiation detection by an infrared thermography method. Fatigue Fract Eng Mater Struct 33(1):12-21

31. Starke P, Walther F, Eifler D (2006) PHYBAL - a new method for lifetime prediction based on strain, temperature and electrical measurements. Int J Fatigue 28(9):1028-1036

32. Kumar A, Torbet CJ, Pollock TM, Wayne Jones J (2010) In situ characterization of fatigue damage evolution in a cast $\mathrm{Al}$ alloy via nonlinear ultrasonic measurements. Acta Mater 58(6):2143-2154

33. Funk M, Eberl C (2011) Investigations on the fatigue behavior of nanocrystalline metals. Key Eng Mater 465:207-210

34. Zimmermann M (2012) Diversity of damage evolution during cyclic loading at very high numbers of cycles. Int Mater Rev 57:2. doi: 10.1179/1743280411Y.0000000005

35. Koster M, Wagner G, Eifler D (2010) Cyclic deformation behavior of a medium carbon steel in the VHCF regime. Proc Eng 2(1): 2189-2197

36. Rabe U, Helfen T, Weikert M, Hirsekorn S, Herrmann HG, Boller C, Backe D, Balle F, Eifler D (2012) Nonlinear ultrasonic testing of carbon fibre reinforced plastics in the very high cycle fatigue regime. Acoust Soc Am Proc Meetings Acoust 16:1

37. Thompson AW, Backofen WA (1971) The effect of grain size on fatigue. Acta Metall 19(7):597-606

38. Kennerknecht T (2014) Fatigue of micro molded materials Aluminium Bronze and Yttrium stabilized Zirconia. Dissertation, Karlsruhe Institute of Technology

39. Baumeister G, Okolo B, Rögner J (2008) Microcasting of Al bronze: influence of casting parameters on the microstructure and the mechanical properties. Microsyst Technol 14(9-11):1647-1655

40. Burger S, Eberl C, Siegel A, Ludwig A, Kraft O (2011) A novel high-throughput fatigue testing method for metallic thin films. Sci Technol Adv Mater 12:054202 